\title{
USE OF MICROWAVES FOR THE DETECTION OF CORROSION UNDER INSULATION
}

\author{
R. E. JONES, F. SIMONETTI, M. J. S. LOWE, IMPERIAL COLLEGE, London, UK \\ I. P. BRADLEY, BP Exploration and Production Company, Sunbury on Thames, UK
}

\begin{abstract}
Corrosion Under Insulation (CUI) is a significant cause of pipeline failure in the oil and gas industry. This paper describes the initial developmental stages of a technique which uses the structure of an insulated pipeline as a coaxial waveguide to support the propagation of microwaves. These microwaves are used to inspect the insulation layer for the presence of water, which will indicate the regions of the pipe at risk from CUI.
\end{abstract}

\section{Introduction}

Corrosion under insulation (CUI) can occur on pipelines fitted with thermal insulation. The insulation is usually meant for process reasons, but can also be for personnel protection. In order to protect the insulation from the environment, and to keep it dry, the insulation layer is covered with a layer of metallic weatherproofing cladding and sealed to prevent moisture ingress. This cladding can deteriorate from age or become damaged, allowing the ingress of water into the insulation which allows corrosion of the external pipe surface to initiate. This corrosion can proceed at an accelerated rate due to the high temperature in proximity to the pipe, and can lead to a failure of the pipeline.

Current methods of pipeline inspection, such as guided wave ultrasound, are sensitive only to regions in which corrosion has already initiated and caused a reduction in wall-thickness. Therefore, it would be preferable to be able to detect the presence of water within the insulation, as an early warning of CUI. There are currently some methods of inspecting pipelines for the presence of water, such as thermography and neutron backscatter, but the former has low sensitivity due to the presence of the cladding and the latter has a small inspection area and is therefore impractical for the inspection of entire lengths of pipeline. There exists a requirement for a screening non-destructive examination (NDE) technique to monitor lengths of pipeline for the first ingress of water into the insulation, providing an early warning of the likely occurrence of CUI and prompting remedial action to reseal the cladding, thereby preventing corrosion from initiating.

This paper describes the initial developmental stages of a screening technique to inspect the insulation layer of a pipeline for the presence of water patches, using low-frequency microwaves. Microwaves are seeing increased application in the field of NDE [1-4], however the technique described here is different in that it uses the structure of the pipeline under inspection as a waveguide to support the propagation of the microwave signal.

The basis for our approach is the observation that a clad insulated pipeline will behave as a coaxial waveguide supporting the propagation of electromagnetic waves. The pipe acts as the inner conductor of the coaxial waveguide, and the cladding will act as the outer conductor. The microwaves will propagate down the length of the pipeline within the insulation layer between the two conductors, excited by an antenna inserted into the insulation. If damaged cladding has allowed the ingress of water into the insulation, then the wet insulation will act as an impedance discontinuity, causing a 
partial reflection of the microwave signal. As with pulse-echo ultrasonics, these reflections can be used to detect the presence of water patches, as shown in Figure 1.

The reflection mechanism is governed by impedance contrast within the insulation. The characteristic impedance of a coaxial waveguide is given by the equation,

$$
Z_{0}=\frac{60}{\sqrt{\varepsilon_{r}}} \ln \left(\frac{a}{b}\right)
$$

where $Z_{0}$ is the characteristic impedance of the waveguide, $\varepsilon_{r}$ is the relative permittivity of the dielectric material filling the annular space of the coaxial waveguide, $a$ is the radius of the outer conductor and $b$ is the radius of the inner conductor. For the types of insulation materials that are commonly used industrially, relative pemittivities in the range of $\varepsilon_{r}=2$ to 3 would be expected, as these dielectric materials are effectively transparent to microwave frequencies.

From Equation 1 it can be seen that the impedance is directly related to the relative permittivity of the dielectric material filling the waveguide. If the insulation is homogeneous then the impedance will be uniform along the length of the waveguide, and will not give rise to any reflections. However if water has accumulated within the insulation then the relative permittivity of the wet insulation will be very different to that of the dry insulation, as the relative permittivity of water at microwave frequencies has a relatively high value of approximately 80 . Therefore, a section of wet insulation will act as an impedance discontinuity within the waveguide, giving rise to a reflection of the incident microwave signal, as shown in Figure 1.

\section{Method}

When designing a guided wave inspection system, it is vital that the modes of propagation and their dispersive characteristics are understood. This information can be obtained from the dispersion curves for the modes of propagation in a coaxial waveguide by solving Maxwell's equations, the semianalytical solution to this problem being discussed by Waldron [5]. An example of these dispersion

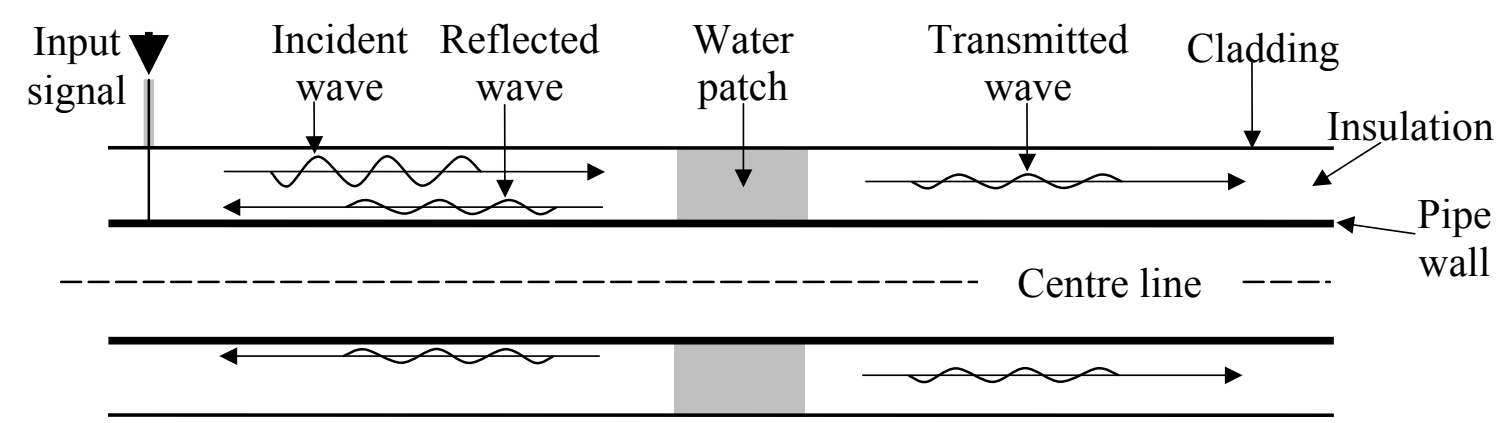

Figure 1. A schematic diagram showing how the pipeline acts as a coaxial waveguide, supporting the propagation of microwaves. A water patch will act as an impedance discontinuity within the waveguide, causing a partial reflection which is used to detect and locate the water patch. 
curves is shown in Figure 2(a), which plots the normalized phase velocity for several of the lowest modes as a function of frequency. Each of the lines represents a separate mode of propagation, and these are labeled according to the mode nomenclature adopted by Waldron. At low frequencies, there is only a single mode propagating, but as the frequency is increased above the cutoff frequency of a particular mode, that mode begins to propagate. It can be seen that the majority of the modes are highly dispersive, with the exception of the mode labeled $\mathrm{TEM}_{00}$, also known as the transmission-line mode, which is non-dispersive at all frequencies.

In order to be able to successfully use guided microwaves for inspection purposes, there are three specific requirements of the mode used to form the signal. The first of these is that the mode should be non-dispersive. The propagation of a dispersive mode leads to wavepacket distortion, which complicates signal interpretation. The second requirement is to maintain mode purity, as the propagation of a signal containing multiple modes, again, complicates signal interpretation. The third requirement is to propagate a signal with as wide a bandwidth as possible, as a wide bandwidth is required for good spatial resolution of waveguide features. The temporal extent of the signal is given by the equation $\Delta t=1 / B$, where $\Delta t$ is the temporal resolution and $B$ is the bandwidth of the signal, calculated using $B=f_{\max }-f_{\min }$. The spatial resolution of features along the axis of the waveguide is directly related to this temporal resolution. Therefore resolution of closely spaced waveguide features is dependant on having a sufficiently wide bandwidth.

In order to determine whether these three requirements can be satisfied by a particular mode of propagation, the dispersion curves were examined for the coaxial waveguides formed by pipeline specifications commonly used in industry. Two of these sets of dispersion curves are displayed in Figure 2. Figure 2(a) shows the dispersion curves for a 6 inch $(152 \mathrm{~mm})$ pipeline with 3 inch $(76 \mathrm{~mm})$ insulation, and Fig 2(b), for a 60 inch $(1524 \mathrm{~mm})$ pipeline with 3 inch insulation. The modes shown in Figure 2 are labeled, either as $\mathrm{TE}_{\mathrm{pq}}$ modes or $\mathrm{TM}_{\mathrm{pq}}$ modes. The $\mathrm{TE}$ and $\mathrm{TM}$ in the mode nomenclature describe two distinct series of modes; Transverse Electric (TE) modes have no axial electric field component, whilst Transverse Magnetic (TM) modes have no axial magnetic field component. The integers $p$ and $q$ describe the field pattern of the mode. The $p$ denotes the number of oscillations of the field pattern around the circumference of the waveguide, whilst $q$ is linked to the number of oscillations radially between the inner and outer conductors.

The TEM $_{00}$ mode represents the ideal mode with which to perform an inspection as it is nondispersive, and so satisfies the first of the three requirements. In order to satisfy the second requirement and maintain mode purity, only frequencies up to the cutoff frequency of the lowest mode, the $\mathrm{TE}_{11}$ mode, can be used. Figure 2 shows that the cutoff frequency of this lowest mode moves towards lower frequencies with increasing pipe diameter. In the 6 inch pipe, the cutoff frequency for the $\mathrm{TE}_{11}$ mode occurs at approximately $400 \mathrm{MHz}$, whilst in the 60 inch pipe, it occurs at approximately $60 \mathrm{MHz}$. In either case, this constraint on the upper frequency limit severely restricts the available bandwidth, especially with larger pipe diameters, resulting in inadequate spatial resolution of waveguide features. 
Dispersion curves for modes in a 6" pipe with 3" insulation

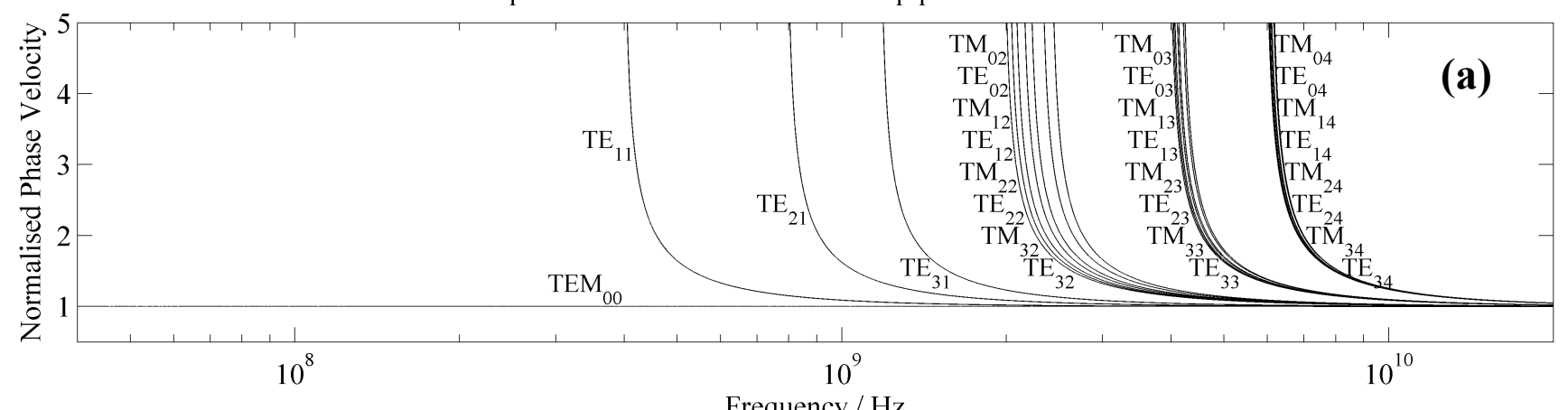

Frequency / Hz

Dispersion curves for modes in a 60" pipe with 3 " insulation

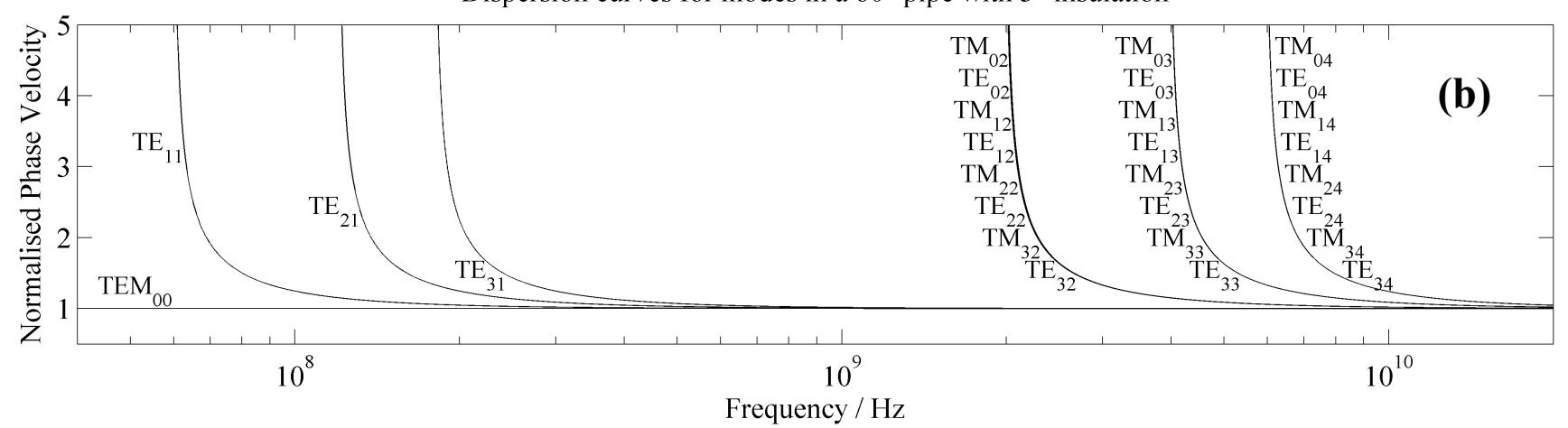

Figure 2. Phase velocity dispersion curves for: (a) 6 inch (152mm) pipe with 3 inch (76mm) insulation, and (b) 60 inch $(1524 \mathrm{~mm})$ pipe with 3 inch insulation. The cutoff frequencies for the TEp1 mode family move towards lower frequencies as the diameter of the pipe is increased. However the cutoff frequencies for the $\mathrm{TM}_{\mathrm{p} 2}$ and $\mathrm{TE}_{\mathrm{p} 2}$ mode families remain relatively constant.

However, if the lowest mode family, the $\mathrm{TE}_{\mathrm{p} 1}$ mode family, can be filtered out, then frequencies up to the cutoff of the next lowest mode, the $\mathrm{TM}_{02}$ mode, can be used. From Figure 2, it is apparent that the cutoff frequency for this mode remains relatively constant at approximately $2 \mathrm{GHz}$, as the pipe diameter is increased. This is because the cutoff frequency of this mode is dictated by the radial distance between the inner and outer conductors, in other words, the insulation thickness, and this is being kept constant at 3 inches. By using frequencies up to the cutoff of the $\mathrm{TM}_{02}$ mode, the available bandwidth would be sufficient for good spatial resolution of waveguide features irrespective of the diameter of the pipe.

For the purpose of filtering out the $\mathrm{TE}_{\mathrm{p} 1}$ mode family, use is made of the fact that the $\mathrm{TEM}_{00}$ mode is axisymmetric and the spurious $\mathrm{TE}_{\mathrm{p} 1}$ modes are non-axisymmetric. This difference in the modes' field patterns can be used as a differentiating factor to enable the spurious modes to be filtered out. Integrating the total field around the circumference of the waveguide would cause the contribution of the $\mathrm{TE}_{\mathrm{p} 1}$ modes to vanish, as their non-axisymmetric field patterns are periodic functions about the circumference. This integration would leave behind only the axisymmetric $\mathrm{TEM}_{00}$ mode, which is ideal for the purpose of inspection. In practice, this integration can be achieved using an antenna array. The array requires two antenna per wavelength of circumferential variation of the field pattern of the highest propagating member of the $\mathrm{TE}_{\mathrm{p} 1}$ mode family. For the 6 inch pipe with 3 inch insulation, the highest propagating mode within the frequency range being used is the $\mathrm{TE}_{41}$ mode, therefore 8 antennas are required and these are arranged in an array around the circumference of the waveguide. This ring-array approach is based on that used in pipe inspection with guided-wave ultrasound [6-8]. 


\section{Experimental Results}

In order to test the feasibility of the technique in practice, two experiments were designed. The first is an experiment on a small coaxial waveguide to assess the achievable signal to noise ratio in the absence of complications due to the propagation of higher order modes. The second experiment involves a coaxial waveguide with dimensions equivalent to a 6 inch pipe with 3 inch insulation, to test the principle of filtering out the higher order modes using an antenna array.

In both experiments a vector network analyzer (VNA) was used to generate the microwave frequency signal. The VNA model used was an Agilent E8361C, with a frequency range from $10 \mathrm{MHz}$ to $67 \mathrm{GHz}$. The VNA sweeps through the input frequency range and records the reflection coefficient from the device under test, the S11 scattering parameter. From this information, a process called Time Domain Reflectometry (TDR) is used to transform the frequency domain data into the time domain, by Fourier analysis. The velocity of propagation is then used to calibrate the time axis to distance, in order to determine the positions of the reflectors.

\section{Small Coaxial Waveguide}

The waveguide for this experiment was formed from two steel pipes with lengths of 6 metres. The inner pipe had an outer diameter of $25.4 \mathrm{~mm}$ and a wall thickness of $2.3 \mathrm{~mm}$, and the outer pipe had an outer diameter of $37.8 \mathrm{~mm}$ and a wall thickness of $2.9 \mathrm{~mm}$. The inner pipe was supported coaxially within the outer by sections of tissue paper wrapped around the inner pipe, at four intervals along its length. Only a single antenna was required in this waveguide, as no higher order modes were propagating in the frequency range being used. The antenna was simply an extension of the inner conductor of the coaxial connector, such that electrical contact was made with the inner pipe. The antenna was positioned a quarter wavelength from a short circuit in order to achieve constructive interference in the desired direction of propagation. The frequency range used in this experiment was from $10 \mathrm{MHz}$ to $1.9 \mathrm{GHz}$. The experimental setup is shown in Figure 3.

Figure 4 displays the results from the small coaxial waveguide experiment. The microwave signal experiences a strong reflection from the antenna at 2.3 metres, which is caused by the impedance mismatch between the feeding cable and the antenna-waveguide system. This means that the majority of the energy incident on the antenna is reflected back into the feeding cable and only a small proportion of the energy is transmitted into the waveguide. There is a smaller peak at 8.3 metres, which occurs 6 metres after the reflection from the antenna. This distance coincides with the length of the waveguide, and the peak is caused by the microwave signal reflecting off the end of the waveguide.

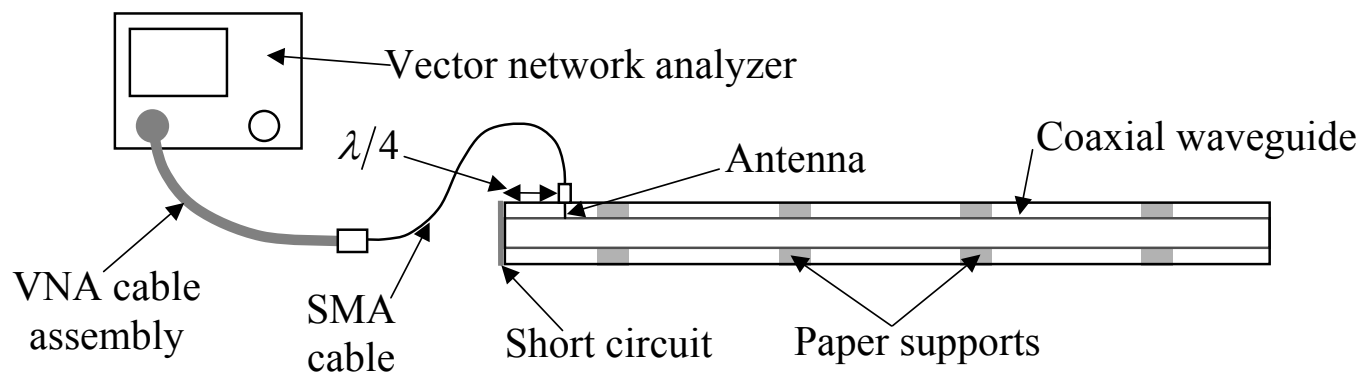

Figure 3. Diagram of the experimental setup used with the small coaxial waveguide. The diameter of the waveguide is sufficiently small that higher order modes do not propagate in the frequency range being used. 
TDR of small coaxial pipes

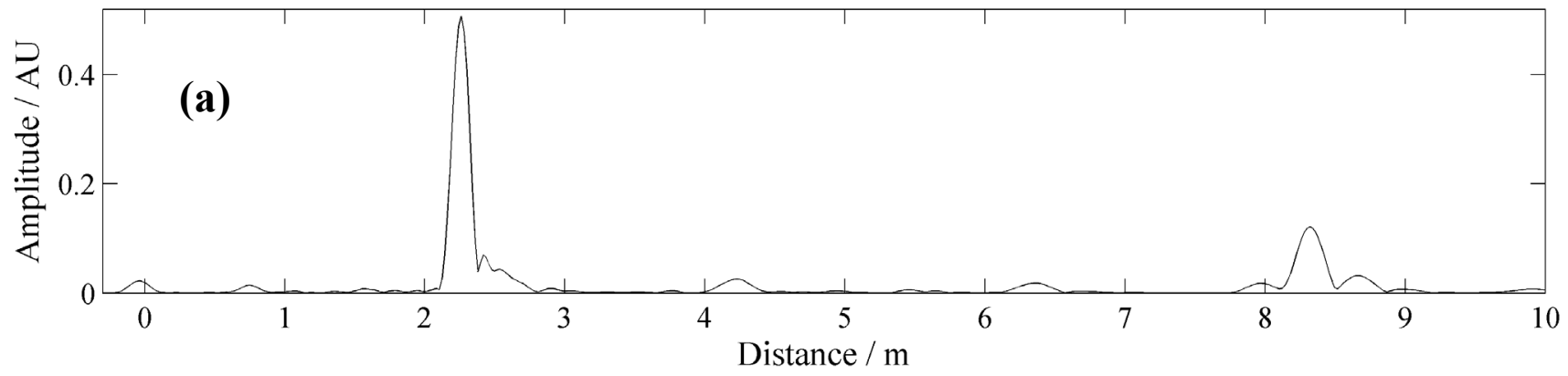

TDR of small coaxial pipes - Scaled in y to the end reflection

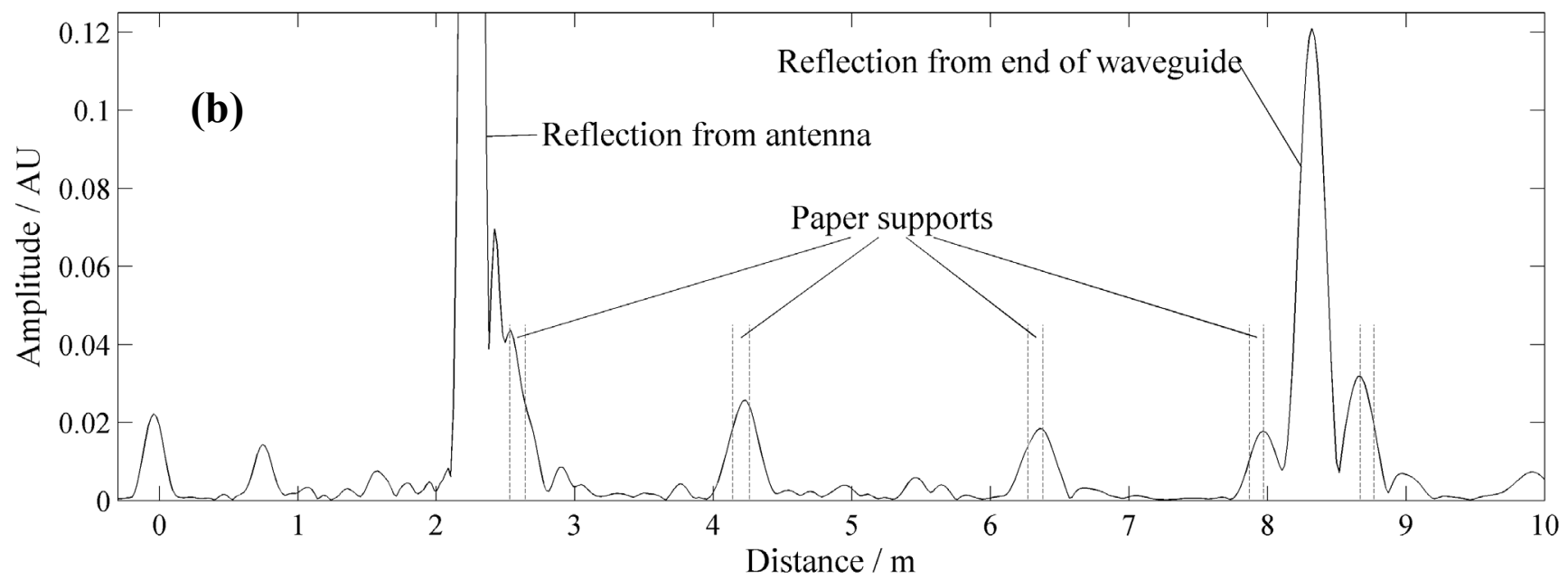

Figure 4. Results recorded from the small coaxial waveguide experiment. (a) Full signal and (b) signal scaled in the y-axis to the amplitude of the end reflection. There is a large reflection from the antenna at $2.3 \mathrm{~m}$, followed by a smaller reflection from the end of the waveguide at $8.3 \mathrm{~m}$. This confirms that the antenna is exciting microwave propagation within the waveguide. The signal shows a very good signal to noise ratio, which enables the reflections from the paper supports to be clearly identified.

Figure 4(b) shows the signal in Figure 4(a) but scaled in the y-axis to the amplitude of the end reflection. The aim of this experiment was to assess the level of signal to noise ratio (SNR) that can be achieved with pure-mode excitation of the TEM $\mathrm{TE}_{00}$ mode. Figure $4(\mathrm{~b})$ shows that the SNR is high, with a value of approximately, $26.3 \mathrm{~dB}$. This SNR allows reflections to be detected from small impedance discontinuities, such as those caused by the presence of the paper supports within the waveguide. The reflections from the supports can be clearly distinguished from the low-level coherent noise, and the positions of the peaks are in good agreement with their measured positions, shown by dashed lines. The ability to detect such small changes in impedance is encouraging for the technique, as water presents a much stronger change in impedance, and consequently should give rise to a stronger reflection.

\section{Pipeline Sized Waveguide}

In order to simulate the waveguide formed by a 6 inch insulated pipeline, industrial ventilation ducting was used. The inner duct had a diameter of $160 \mathrm{~mm}$, and the outer, a diameter of $315 \mathrm{~mm}$, both with a wall thickness of $0.7 \mathrm{~mm}$. Two 3 metre lengths were coupled together to form a 6 metre coaxial waveguide. Once again, the frequency range used in this experiment was from $10 \mathrm{MHz}$, to $1.9 \mathrm{GHz}$. For this particular waveguide geometry, an array with a minimum of 8 antennas was required to suppress 
the $\mathrm{TE}_{\mathrm{p} 1}$ mode family, as explained previously. These antennas were arranged at $45^{\circ}$ intervals around the circumference of the waveguide and positioned a quarter wavelength from a short circuit, for constructive interference. Once again, the antennas were all formed by extending the inner conductor of the coaxial connector until electrical contact was made with the inner conductor of the coaxial waveguide. An 8-way splitter was used to divide the single channel from the VNA into the 8 channels required to feed the antenna array. The splitter also served to recombine the reflected signals, thus removing the non-axisymmetric modes.

Figure 5(a) shows the results recorded from the pipeline sized waveguide experiment. This graph was also scaled in the y-axis to the amplitude of the reflection from the end of the waveguide. The reflection from the antenna occurs at approximately 3 metres, and has a relatively large amplitude due to the impedance mismatch experienced by the microwave signal at the antennas. The peak at 9 metres is due to the reflection of the signal from the end of the waveguide. In the region between $3 \mathrm{~m}$ and $8 \mathrm{~m}$, there is a relatively high level of coherent noise, and this is due to reverberations within the feeding cables, which in turn, are due to the impedance mismatch at the antennas. Therefore by addressing the issue of the impedance mismatch, the level of coherent noise will be reduced. However in order to observe the response of this system to the presence of a water defect in the waveguide, a bottle filled with water was used to represent a patch of wet insulation. The bottle had a length of $200 \mathrm{~mm}$ and a diameter of $50 \mathrm{~mm}$, which equates to $3 \%$ of the cross-sectional area of the waveguide. The bottle was positioned 1 metre from the end of the waveguide and was aligned axially. In Figure 5(a), it can be

Signals with and without the presence of a water defect

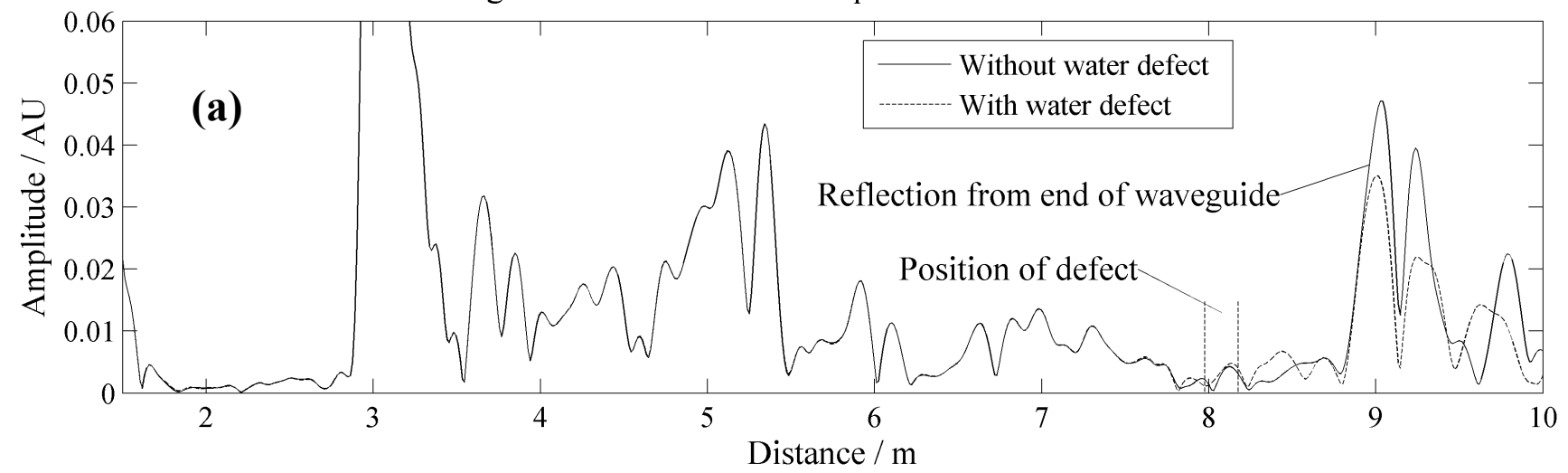

Signal from a water defect produced from baseline subtraction

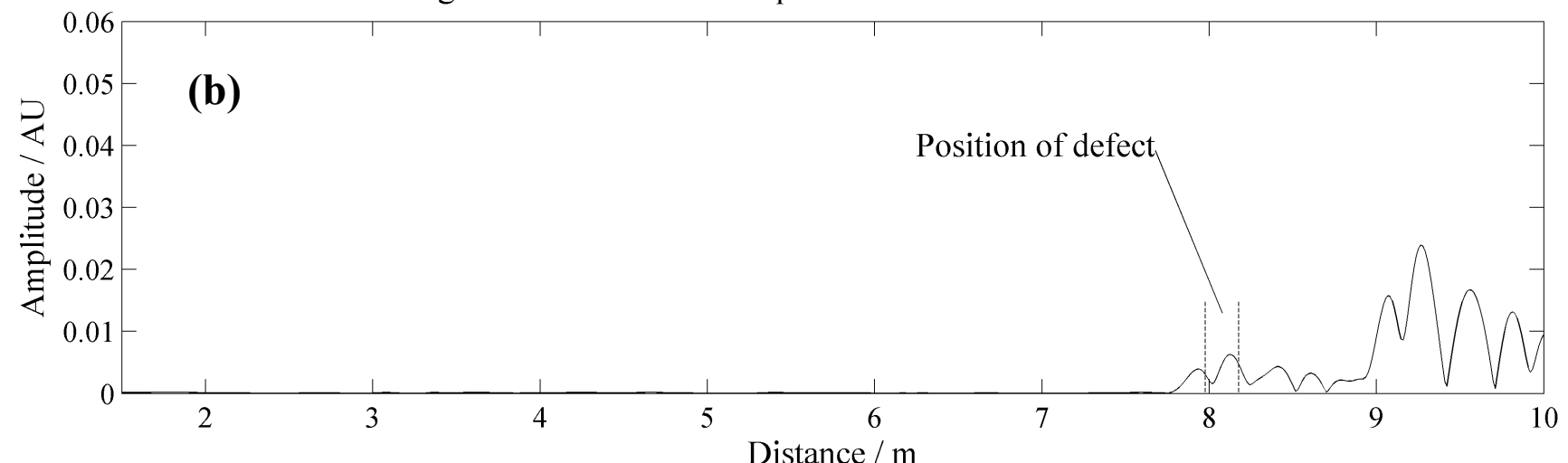

Figure 5. Results recorded from the pipeline sized waveguide experiment. (a) A water defect covering 3\% of the crosssectional area was introduced into the waveguide. The presence of water within the waveguide significantly reduces the amplitude of the end reflection, due to water's attenuation of microwave frequencies. (b) A baseline subtraction technique was used to isolate the response due to the water defect. The residual signal shows that this defect has been clearly detected. 
seen that the presence of the water defect significantly reduces the amplitude of the reflection from the end of the waveguide. This is due to the attenuation of the microwave signal by the water within the waveguide. In order to isolate the response due to the water patch, a baseline subtraction technique was used, with a baseline signal recorded before inserting the water bottle. This residual signal can be seen in Figure 5(b). The presence of the water defect is clearly detected despite the relatively small crosssectional area. Close inspection of Figure 5(a) reveals that the signal changes at the location at which the defect is positioned. If the coherent noise was eliminated, the reflection at this point, due to the defect, could be detected. This method of inspection would be the ideal approach, as the technique does not require a baseline and so is not affected by problems associated with baseline instability. In addition, the direct detection of reflections allows the location of a defect to be accurately determined. However, this approach does require a high SNR, such as that achieved in the small coaxial waveguide experiment.

\section{Conclusions}

The initial developmental stages of a guided microwave technique to inspect pipeline insulation for the presence of water, have been described here.

The results from the small coaxial waveguide experiment show that a low level of coherent noise can be obtained, if excitation of only the ideal inspection mode (the non dispersive, axisymmetric TEM $_{00}$ mode) can be achieved. For the inspection of larger, more realistic, pipe dimensions, the presence of higher-order spurious modes complicates pure-mode excitation in the frequency range of interest. It has been demonstrated that these issues can be overcome, and pure-mode excitation can be achieved through the use of an antenna array.

Initial experimental results have shown that the technique can achieve high sensitivity to water patches, as a patch as small as 3\% of the insulation cross section has been successfully detected. This is due to the high attenuation of microwaves in water. The ideal approach would be to directly detect the reflections from water patches without using baseline subtraction. This would require improving the signal to noise ratio, by addressing the issue of the impedance mismatch at the antennas. Eliminating the impedance mismatch will prevent signal reverberation in the feeding cable system, thereby reducing the coherent noise level.

\section{Acknowledgements}

This work is supported by BP and by the UK Engineering and Physical Sciences Research Council (EPSRC) through the UK Research Centre in NDE.

\section{References}

1. R. Zoughi and S. Bakhtiari, IEEE Trans. Instrum. Meas., 39, 1059-1063 (1990).

2. C. Yeh and R. Zoughi, IEEE Trans. Instrum. Meas., 43, 719-725 (1994).

3. S. Ganchev, R. Zoughi et al, IEEE Trans. Microw. Theory Tech., 42, 18-24 (1994).

4. K. Bois, A. Benally and R. Zoughi, IEEE Trans. on Instrum. Meas., 49, 49-55 (2000).

5. R. A. Waldron, Theory of Guided Electromagnetic Waves, Van Nostrand-Reinhold, New York, 1970, Chapter 4.

6. M. J. S. Lowe, D. N. Alleyne and P. Cawley, Ultrasonics, 36, 147-154 (1998).

7. D. N. Alleyne, M. J. S. Lowe, and P. Cawley, J. Appl. Mech., 65, 635-641 (1998).

8. M. J. S. Lowe, D. N. Alleyne and P. Cawley, J. Appl. Mech., 65, 649-656 (1998). 